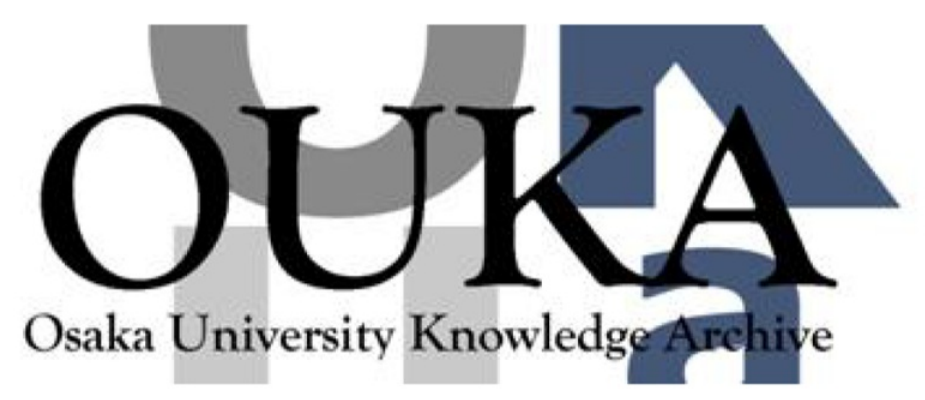

\begin{tabular}{|c|l|}
\hline Title & $\begin{array}{l}\text { A conservative momentum exchange algorithm for } \\
\text { interaction problem between fluid and } \\
\text { deformable particles }\end{array}$ \\
\hline Author(s) & $\begin{array}{l}\text { Takeuchi, Shintaro; Yuki, Yoshihiko; Ueyama, } \\
\text { Atsushi et al. }\end{array}$ \\
\hline Citation & $\begin{array}{l}\text { International Journal for Numerical Methods in } \\
\text { Fluids. 64(10 12) p. 1084-p. 1101 }\end{array}$ \\
\hline Issue Date & $2010-02-16$ \\
\hline oaire:version & AM \\
\hline URL & https://hdl. handle. net/11094/57217 \\
\hline rights & \\
\hline Note & \\
\hline
\end{tabular}

Osaka University Knowledge Archive : OUKA

https://ir. Library. osaka-u. ac. jp/

Osaka University 


\title{
A Conservative Momentum Exchange Algorithm for Interaction Problem between Fluid and Deformable Particles
}

\author{
Shintaro Takeuchi ${ }^{1}$, Yoshihiko Yuki ${ }^{2}$, Atsushi Ueyama ${ }^{2}$ and Takeo Kajishima ${ }^{2}$ \\ ${ }^{1}$ Department of Mechanical Engineering, The University of Tokyo, \\ 7-3-1 Hongo, Bunkyo, Tokyo 113-8656, Japan \\ ${ }^{2}$ Department of Mechanical Engineering, Osaka University \\ 2-1 Yamada-oka, Suita-city, Osaka 565-0871 Japan
}

\begin{abstract}
A new method for studying interaction between elastic object and a fluid is developed. The fluid phase including solid interface is solved by our immersed boundary method of body-force type (Kajishima et al., JSME Int. J. Ser. B, 44-4, pp.526-535, 2001; Kajishima \& Takiguchi, Int. J. Heat and Fluid Flow 23(5), pp.639-646, 2002). The method has enabled simulation of interaction problem between a fluid and rigid spherical particles of a total number of $O\left(10^{3}\right)$ by an efficient momentum-exchange technique through volume-averaged velocity field of multiple phases. In the present study, the fluid-solid interaction force is incorporated into FEM. This process is done by a superposition of the hydrodynamic force field with the solid internal force field. The uniqueness of the present approach lies in the implementation of the inter-phase momentum exchange through the distributed force field shared by both Eulerian and moving Lagrangian references. The applicability of the present method is demonstrated in two 2-D flow fields including a large number of elastic particles. The effects of particle geometry and elasticity are investigated. The results suggest that particles' neutral geometry and deformability exhibit strong correlation to rotating motions and clustering behaviours of the particles in the fluid flow.
\end{abstract}

\section{Keywords}

Fluid-solid interaction, Deformability, Elastic particle, Clustering behaviour, Direct numerical simulation 


\section{Introduction}

Interaction problem between fluid and solid object constitutes one of the most challenging subjects in the engineering field, particularly when involving large density ratio and/or different stress-strain relations. Despite the broad scope of applications ranging from biology (e.g. biological cells [1] or vesicles [2] in a viscous shear flow) to large scale structures (e.g. parachute aerodynamics [3, 4] and flutter of air-plane wings [5]), modelling of fluid-solid interaction has been still in development due to its multi-physics nature over ranges of time- and length-scales. Numerical simulation is suitable for handling a wider range of fluid-structure properties that are inaccessible by experiments simultaneously and non-invasively.

There are currently several major approaches for simulation of fluid-structure interaction. The examples include arbitrary Lagrangian-Eulerian (ALE) method[6] in body-fit curvilinear coordinate system and the DSD/ST procedure [7, 8] with finite-element unstructured mesh. Also, noticeable contributions are found to the development of numerical methods on fixed (time-independent) rectilinear coordinate systems, such as immersed boundary (IB) method [9] and ficticious domain method [10].

In the methods with the body-fit coordinates/unstructured-mesh, high resolutions near the boundary layer is expected as the flow is resolved along the moving/deforming object surface. The body-fit approach has been well-established and widely employed for problems involving moving rigid objects in 2-D [11] and 3-D [12] and deforming objects [13, 14, 15, 16, 17]. However, although local re-meshing techniques were developed and successfully tested in [18], most of the time the whole computational domain is re-meshed as the object moves/deforms, which is computationally intensive. Also, perhaps because the approach has some difficulties to such systems as involving an object with complex geometry or freely-moving polysized particles (causing meshing over non-simply-connected regions), not many examples are reported for a large-population of fluid-solid suspension problems (e.g. [18, 19]). In addition, the body-fit simulation technique may not be very advantageous for studying turbulence statistics and vortical structures (shed from particles and bubbles) at a uniform scale (resolution).

In the fixed-mesh techniques, on the other hand, the computational mesh is generally a structured mesh, often rectangular or even uniform mesh. The mesh shape does not fit the object's shape. Therefore, the particular difficulty lies in the momentum exchange at the moving interface. For this purpose, some specially designed force term is incorporated into the dynamics of the two phases in order to incorporate the effect of the fluid-solid interface and/or to locally enforce the solution to the adequate constraint. This approach enables investigation of flow characteristics with a simple numerical treatment and relatively low computational load. For these reasons, the fixed-grid approach has been increasingly adopted for simulating multi-phase problems, and several models are readily to be available for studying fundamentals of a large-scale fluid-solid suspension system (e.g. [20]). 
Among the several methods existing, the IB approach originally proposed by Peskin [9, 21] is probably the most widely adopted method. The main idea of his method is to represent fluid-solid boundary with Lagrangian markers located at the object surface immersed in a fluid. The fluid and solid phases share the physical properties at the "immersed" boundary by mutual interpolation between the Eulerian and Lagrangian references via a pseudo delta function as a weight function. The method has demonstrated its versatileness through applications to a number of problems including blood flow in a heart [9], and deformable biological cells experiencing shear [23] and motion of aquatic animals [22]. The IB method has also inspired many researchers to propose a number of improved methods. For example, to facilitate the application to medical problems, Zhang and co-workers $[24,25]$ proposed (in their immersed finite element method) a new kernel function, instead of the pseudo delta function, for determining the cut-off region around the interface on a non-uniform mesh system. Also, the immersed interface treatment [26, 27, 28] improves the sharpness of the interface by incorporating the jump in the stress and velocities across the interface.

We have independently developed another efficient fluid-structure coupling technique with a conservative momentum exchange (CMX) algorithm for solid objects immersed in a fluid [29, 30]. In the method, no interpolation is performed between the phases, as the fixed rectilinear grid is shared with both fluid and solid for representing velocity fields of the two phases. The interaction force, modelled as a volume force, is given to the both phases with the opposite signs on the same (shared) grid point. Therefore, the method ensures no momentum leakage between the phases. This approach enabled high speed computation compared to the above IB-based/ficticious domain methods [31], and this method has been applied to direct numerical simulation (DNS) of particle-laden turbulence of a total number of $O\left(10^{3}\right)$ spherical particles [30]. Nishiura et al. [32] combined our method with a discrete particle method [33] for solving a sedimentation process involving interparticle collisions in a $10^{5}$-particle system.

When the solid particles exhibit deformable nature, the deformability of the particles may add more special characteristics to the behaviour of the fluid-solid mixture. In the past decades, the above studies of the dynamics of the deformable membrane structure in a viscous fluid have been driven by the strong interests in biological applications. However, the meso-scopic flow structures induced by a large population of deformable particles have not been studied.

This paper aims to present a new concept of numerical approach for solving interaction problems between fluid and a large number of elastic solid objects. The fluid motion is solved on a fixed grid by finite difference (FD) method, and the solid deformation is solved by a finite element (FE) approach, together with the two-way coupling through the above momentum-exchange algorithm to handle a large number of particles efficiently. A unique coupling idea of the mixed FD-FE approach and the momentum exchange 


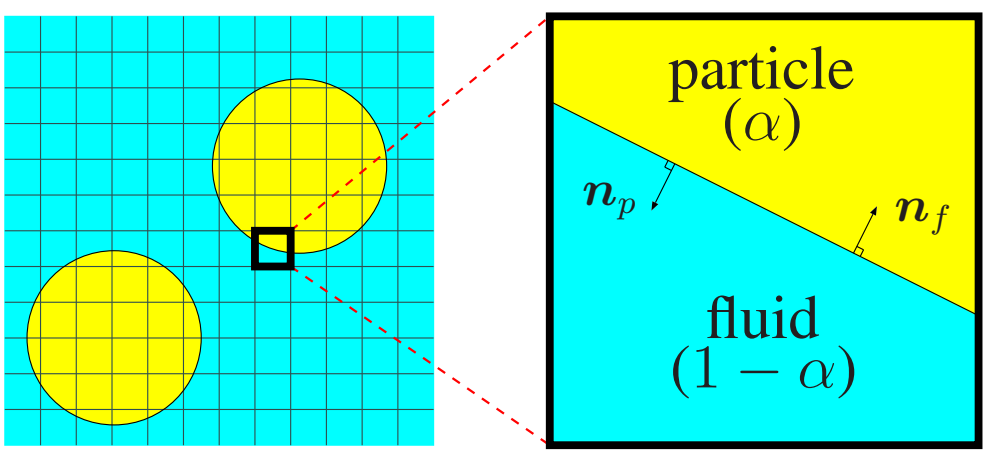

Figure 1: Schematic of a interface between fluid and particle phases cutting through a computational cell. Left: part of the computational domain (uniform Cartesian grid) filled with the fluid-phase and dispersed particles, right: the definition of solid volumetric fraction $\alpha$ and interfacial normal vectors

algorithm is proposed, and the applicability of the coupling algorithm is demonstrated in a fluid-solid suspension in a vertical channel. Effects of the neutral geometry and deformability of the particles on the clustering behaviours are studied.

\section{Numerical Methods}

In the fluid computational cells partially occupied by the solid object, the fluid-solid interaction is solved by a conservative momentum-exchange algorithm for solid object immersed in an underlying fluid, assuming that the solid object behaves like a rigid body at every instance. The momentum-exchange algorithm, originally proposed by Kajishima and co-researchers [29, 30], is briefly summarised in the following.

\subsection{Fluid - solid interaction and numerical implementation}

A velocity field $\boldsymbol{u}$ is established through volume-averaging the local fluid velocity $\boldsymbol{u}_{f}$ and the local solid phase velocity $\boldsymbol{u}_{p}$ in each computational cell:

$$
\boldsymbol{u}=(1-\alpha) \boldsymbol{u}_{f}+\alpha \boldsymbol{u}_{p}
$$

where $\alpha(0 \leq \alpha \leq 1)$ is the local solid volumetric fraction in the cell, as illustrated in Fig.1. Note that, for rigid object, $\boldsymbol{u}_{p}$ is decomposed into translating component $\boldsymbol{v}_{p}$ and rotating component $\boldsymbol{\omega}_{p} \times \boldsymbol{r}$, where $\boldsymbol{\omega}_{p}$ is angular velocity and $\boldsymbol{r}$ is a vector from object centre. According to the continuity of the velocity in the normal direction to the interface, $\boldsymbol{u}$ satisfies the equation of continuity $\nabla \cdot \boldsymbol{u}=0$, suggesting that the velocity field $\boldsymbol{u}$ could be treated as a single continuum. The interaction between fluid and solid is solved 
in the following procedure. Assuming that the whole computational domain is filled with a continuum of a uniform density, the basic equation is:

$$
\begin{aligned}
\frac{\partial \boldsymbol{u}}{\partial t} & =-\nabla p+\boldsymbol{H}+\boldsymbol{f}_{I} \\
\boldsymbol{H} & =-\boldsymbol{u} \cdot \nabla \boldsymbol{u}+\frac{1}{\rho_{f}} \nabla \cdot\left[\mu_{f}\left(\nabla \boldsymbol{u}+(\nabla \boldsymbol{u})^{T}\right)\right],
\end{aligned}
$$

where $p$ pressure, $\rho_{f}$ density, $\mu_{f}$ viscous coefficient of the fluid. The interaction force $\rho_{f} \boldsymbol{f}_{I}$ (per unit volume) accounts for the effect of the solid object on the fluid phase in the interface cells and inside the object.

The above equations are time-integrated apparently by the same procedure as the SMAC methods [34] (with the 2nd-order Adams-Bashforth method in Eq.(6)):

$$
\begin{aligned}
& \boldsymbol{u}^{n+1}=\widehat{\boldsymbol{u}}+\Delta t \boldsymbol{f}_{I} \\
& p^{n+1}=p^{n}+\phi \\
& \widehat{\boldsymbol{u}}=\boldsymbol{u}^{*}-\Delta t \nabla \phi, \\
& \boldsymbol{u}^{*}=\boldsymbol{u}^{n}-\Delta t \nabla p^{n}+\Delta t \frac{3 \boldsymbol{H}^{n}-\boldsymbol{H}^{n-1}}{2}, \\
& \nabla^{2} \phi=\frac{\nabla \cdot \boldsymbol{u}^{*}}{\Delta t}, \\
& \boldsymbol{f}_{I}=\frac{\alpha\left(\boldsymbol{u}_{p}-\widehat{\boldsymbol{u}}\right)}{\Delta t}
\end{aligned}
$$

and $\Delta t$ is a time increment. The superscripts indicate time levels. Note that in a cell fully occupied by the solid ( $\alpha=1$ and $\boldsymbol{u}=\boldsymbol{u}_{p}$ ) Eq.(3) reduces to $\boldsymbol{u}^{n+1}=\boldsymbol{u}_{p}$, while in the fluid cells ( $\alpha=0$ and $\boldsymbol{u}=\boldsymbol{u}_{f}$ ) Eqs.(3) (7) coincide with a procedure for a single-phase Navier-Stokes solver (SMAC method) because of $\boldsymbol{f}_{I}=0$. Therefore, the interaction force $\rho_{f} \boldsymbol{f}_{I}$ per unit volume, given at the fluid-solid interface, works to enforce the no-slip condition in the mixture field.

The second order central finite difference is adopted for the terms in Eq.(6). The Poisson equation of Eq.(7) is, again, discretised by the 2nd order central finite difference, and it is solved by the successive over-relaxation (SOR) method in conjunction with the residual cutting method [35].

Solid volumetric fraction $\alpha$ in Eq.(8) needs to be evaluated in each computational cell. For simple geometries such as spheres, $\alpha$ could be determined by a formula, but the computational load associated with this process is fairly significant. An efficient algorithm for digitising the fluid-solid interface is necessary for a deformable object of arbitrary geometry. In the present study, a surface-digitiser of hyperbolic-tangent type $[31,36]$ is used to approximate solid volume fraction. The method has been proved to be highly efficient (even for computing a time-dependent volume fraction for moving/deforming object). 
The particles undergo the same interaction force and the moment of the force with the opposite sign, and time-advancement of the solid phase is completed by integrating the forces over the volume of the particle:

$$
\begin{aligned}
& \frac{d\left(m_{p} \boldsymbol{v}_{p}\right)}{d t}=-\int_{V_{p}} \rho_{f} \boldsymbol{f}_{I} d V \\
& \frac{d\left(\boldsymbol{I}_{p} \cdot \boldsymbol{\omega}_{p}\right)}{d t}=-\int_{V_{p}} \rho_{f} \boldsymbol{r} \times \boldsymbol{f}_{I} d V
\end{aligned}
$$

where $m_{p}$ is mass of the object, $\boldsymbol{I}_{p}$ inertia tensor and $V_{p}$ region enclosing the object. Eqs.(9) and (10) are time-updated by the 2nd order Adams-Bashforth method.

The use of the same interaction force for both phases at the interface cells guarantees the conservation of the momentum between the phases. The integration region in Eqs.(9) and (10) does not necessarily coincide with the particle volume, but a volume enveloping the particle is allowed since $\boldsymbol{f}_{I}$ vanishes outside the solid occupation region. This margined-volume integration of $\boldsymbol{f}_{I}$ (instead of surface integration of the surface forces) considerably reduces the computational load [31], which is the strong advantage of this method. The above method has been validated through the predictions of the drag forces acting on a single spherical particle fixed in space [29] together with the vortex shedding in the wake of a particle, for a range of Reynolds numbers [31]. The method has been applied to direct numerical simulation (DNS) of turbulence induced by $O\left(10^{3}\right)$ spherical particles [30] and particle clustering in a sedimentation process populating $10^{5}$ spherical particles [32].

In the subsequent sections, coupling of CMX algorithm and a finite element formulation is described. The algorithm is found to show good compatibility with FEM, and the transplantation is carried out without any essential modification on the existing computer code developed for studying the above large population of solid suspension problems [29, 30].

\subsection{Finite element procedure and coupling with the momentum-exchange algo- rithm}

Coupling with a standard finite element formulation (for solid deformation) $[37,38]$ of a total Lagrangian form:

$$
M \ddot{z}+Q=F
$$

has been developed [39, 40] with a St.Venant-Kirchhoff material for satisfying solid incompressibility. Here, $\boldsymbol{M}$ is global mass matrix, $\ddot{z}$ acceleration, $\boldsymbol{Q}$ internal force vector and $\boldsymbol{F}$ external force vector. The above equation is time-integrated by the Newmark- $\beta$ method. A coupling test of vibrating beam in a uniform flow has shown that the time history of the deflection is in reasonable agreement with the result 
obtained by a monolithic strong-coupling approach by Ishihara \& Yoshimura [41] (Fig.19 of the literature) with the maximum error of $1.9 \%$ at the largest amplitude.

However, in our preliminary case employing 128 elastic particles falling in a fluid (using SX-8R of Osaka University, a vector-type computer with shared-memory), it was found that a considerable amount of overall computational time (more than 90\%) is consumed for the convergence computation of solid deformations (due to relatively lower compatibility for the solid mechanics solver with the computer architecture, while the fluid routines exhibited the optimised vectorization performance).

In the present work, therefore, we determined to reduce to a system with a linear elastic material obeying Hooke's law (under plane-strain condition) and a simplified formulation based on the infinitesimal-strain assumption:

$$
M \ddot{z}+K \boldsymbol{z}=\boldsymbol{F}
$$

to perform faster computation, within a feasible computational time, with accommodating elastic particles of total number $O\left(10^{2}\right)$ for studying the effect of deformability of the particles on the clustering behaviour.

Incorporation of a damping term is not essential in the present method $[39,40]$. However, again, for the purpose of the present study (proving that the proposed coupling method surely works), damping is incorporated to perform stable and reasonably-fast computation. Damping model should account for some non-linear mechanics of the material to predict the dynamic behaviours of the object, but, generally, it is difficult to identify damping properties of each material. In the present study, a simple viscous damping with no history-effect is assumed, and the well-established Rayleigh damping coefficients [42] are employed.

Our FD-based momentum-exchange algorithm is coupled with the FEM by incorporating $\boldsymbol{f}_{I}$ into the finite-element formulation as an external force, which is briefly explained in the following. The coupling procedure assumes that the deformable object moves sufficiently slow to allow the fluid to have enough time to relax within a single time increment. A purely theoretical estimate of this assumption is presented in Appendix A.

To facilitate the computation of a multi-body system, the deformations of each body are described on the non-inertial frame fixed to each object. For calculating the components in $f_{I}$, objects' local velocities (including internal displacement velocities) must be considered in $\boldsymbol{u}_{p}$. Instantaneous translating and angular velocities are added with the non-inertial displacement velocities $\dot{z}$. The inertial velocities on the object nodes are expressed as:

$$
\boldsymbol{u}_{p}=\boldsymbol{v}_{p}+\boldsymbol{\omega}_{p} \times \boldsymbol{r}+\boldsymbol{R} \dot{\boldsymbol{z}}
$$

where $\boldsymbol{R}$ is a rotation matrix representing the orientation of body with respect to the initial orientation. 
The external force $\boldsymbol{F}$ on the solid body includes the interaction force $\left(-\rho_{f} \boldsymbol{f}_{I}\right)$ assembled over the finite elements. Eq.(11) is time-integrated by the Newmark- $\beta$ method, and the resultant $\dot{z}$ is substituted into Eq.(12). Note that the motions of the fluid (by Adams-Bashforth method) and particles (by Newmark- $\beta$ method) are time-updated alternatively.

As will be detailed again, the time-averaged displacement of gravity centre due to the particle deformation are found to be negligible throughout the simulations. Therefore, the superposition of the deformation velocity is reasonably accepted, and, in turn, so is the mixed FD-FE approach coupled with the momentumexchange algorithm for solving solids suspension problem within a feasible computational time.

\section{Particle-laden Flow: Elastic Particles in an Up-current Flow}

When the solid particles have deformability, the solids suspension may exhibit some different features from the behaviours of the rigid particles. Here, the effects of neutral geometry (non-circularity) and deformability of the particles on a two-dimensional suspension flow and clusterisation of particles are studied.

\subsection{Numerical Conditions}

Ellipticity $n$ (ratio of the major to the minor axes) and Young's modulus $E$ are the major parameters for non-circularity and deformability, respectively. Hereafter, Young's modulus is normalised by the reference pressure of the fluid and denoted by $E^{*}$.

In the present study, particles of $n=1$ (circular particle) and 1.2 are employed. The elliptic particle has the equivalent volume as the circular particle of diameter $D_{p}$. Normalised Young's modulus $E^{*}$ is varied from 1 to 100 . For the material of $E^{*}=100$, the typical order of the hydrodynamic force was found to be too weak to cause any visible displacements on the object in the present study; the hydrodynamic force rather works for translation and rotation of the object than deformation of the object. Therefore, the particles of $E^{*}=100$ can be regarded as rigid case.

Figure 2 illustrates a schematic of the flow field and a initial particle arrangement for an elliptic case. Periodic boundary condition is assumed for both directions. Initial arrangement of the particles allows some random deviations (maximum deviation is $D_{p} / 2$ ) from the uniformly-arranged positions in both directions. Also, initially, the particles are randomly orientated. The particles are released from rest into a fluid, and accelerated by gravity in $-y$ direction. A constant pressure gradient is induced (in the vertical upward direction) to balance with the steady drag forces on the rigid particles of diameter $D_{p}$. The spatial resolution is $D_{p} / \Delta=20$, where $\Delta$ is grid spacing. Reynolds number $R e$, defined with $D_{p}$ and the terminal velocity $U_{0}$ of a single circular particle (rigid), is set to 200. Our previous study shows that, at this Reynolds number, the vortical discharge in the wake is reproduced at the above spatial resolution [31]. 


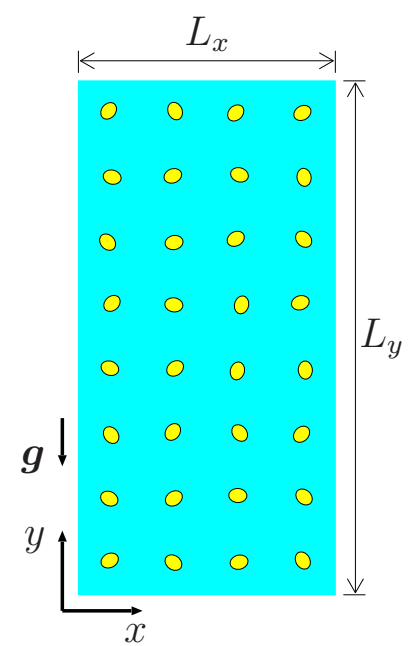

Figure 2: Schematic of the computational domain and an initial particle arrangement (for elliptic case).
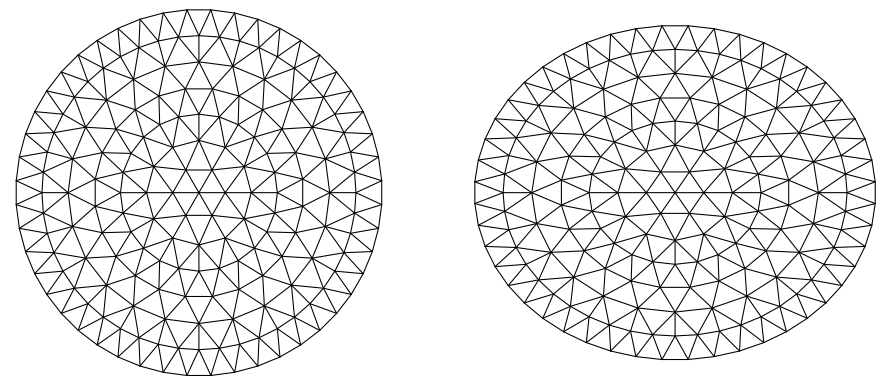

Figure 3: Unstructured grid of elastic particle. Neutral shapes for circular (left) and elliptic (right) particles.

The unstructured grids of the circular and elliptic particles are shown in Fig.3. For both geometry, the number of elements and nodes are 324 and 187, respectively. Our preliminary study shows that the mean deviation of the gravity centre is well below $10^{-3} D_{p}$ throughout the simulations. The maximum deviation of inertia tensor is found to be $2 \%$ from the initial value.

Total numbers of particles used are 32 and 128 (labelled Case 1 and 2, respectively). The bulk solid volume fraction is $0.48 \%$ for both cases, and little effect of collisions on the flow structures is observed. Nevertheless, inter-particle collisions take place accidentally once in about 100 unit-times, and the impact of the colliding event may cause violent disturbance on the flow field. In the present study, an inter-particle repulsive potential same as Glowinski et al. [10] and Uhlmann [43] is introduced to isolate the effect of collisions on the flow structure.

Other numerical conditions are summarised in Table 1 together with the above conditions.

\subsection{Results and Discussion}

Figure 4 shows some snapshots of the flow fields, in a developing stage, including 32 circular and elliptic particles of $E^{*}=1$. Figures 4(a) and 4(b) show distributions of the particles (white) in the domain and shed 


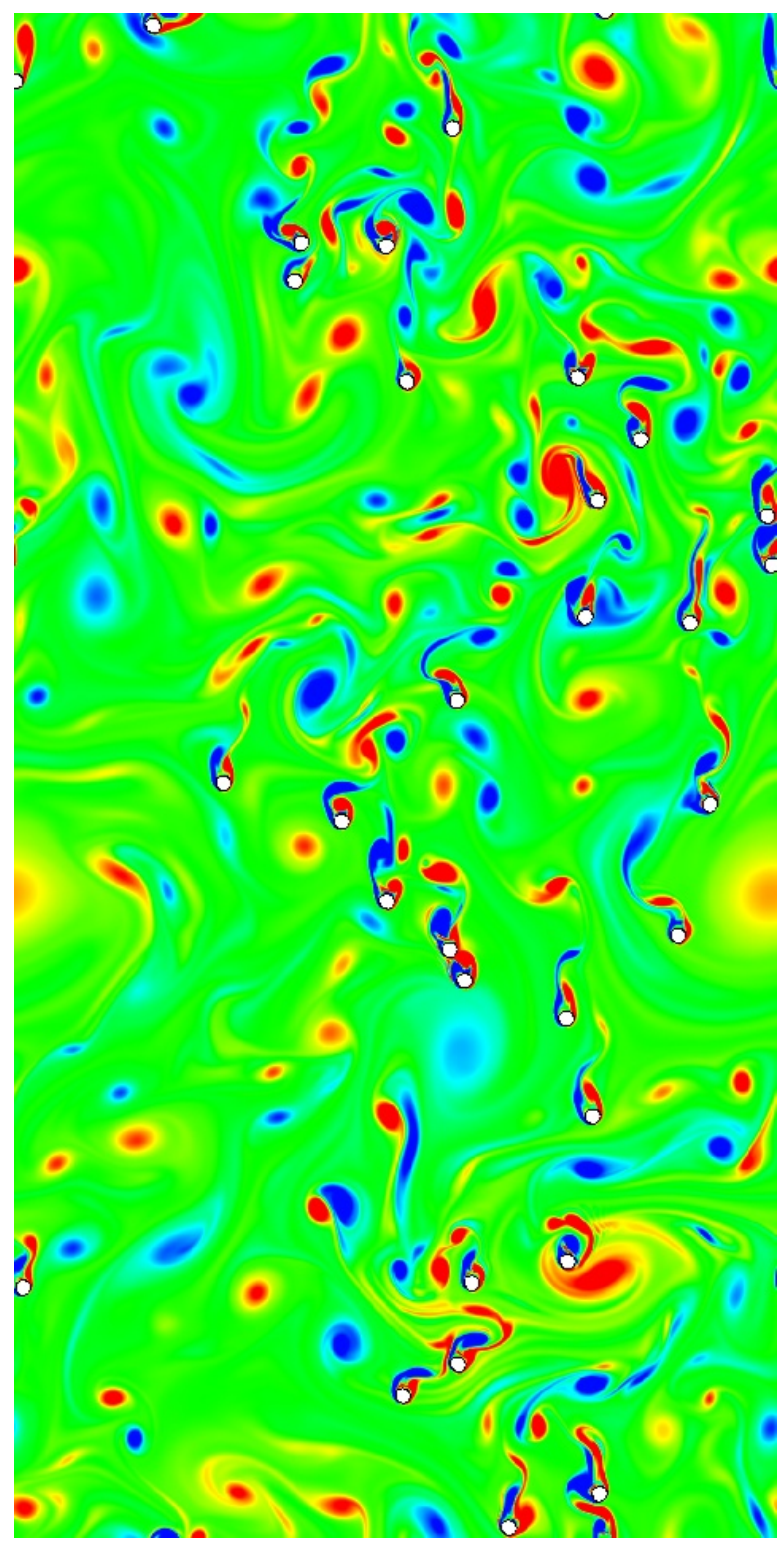

(a) Vortical structures for Case 1 with $E^{*}=1$ and $n=$ 1

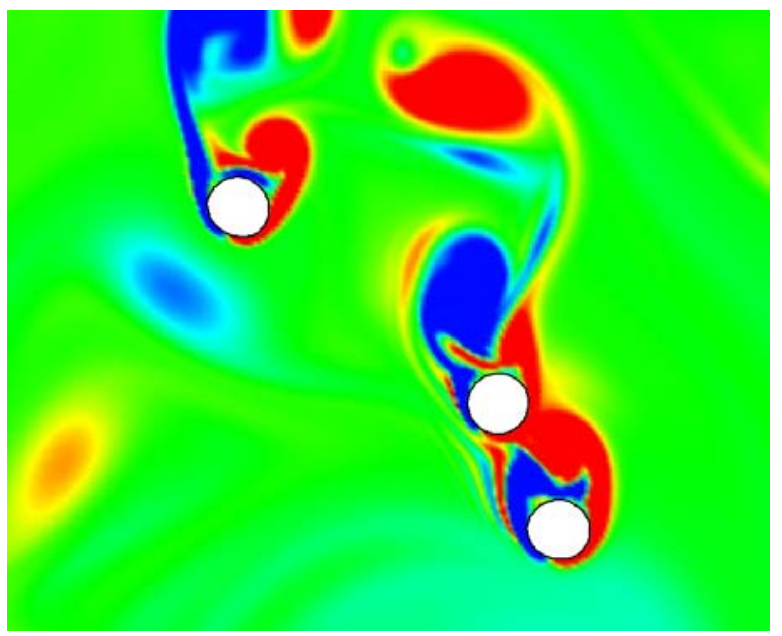

(c) A close-up image of (a)

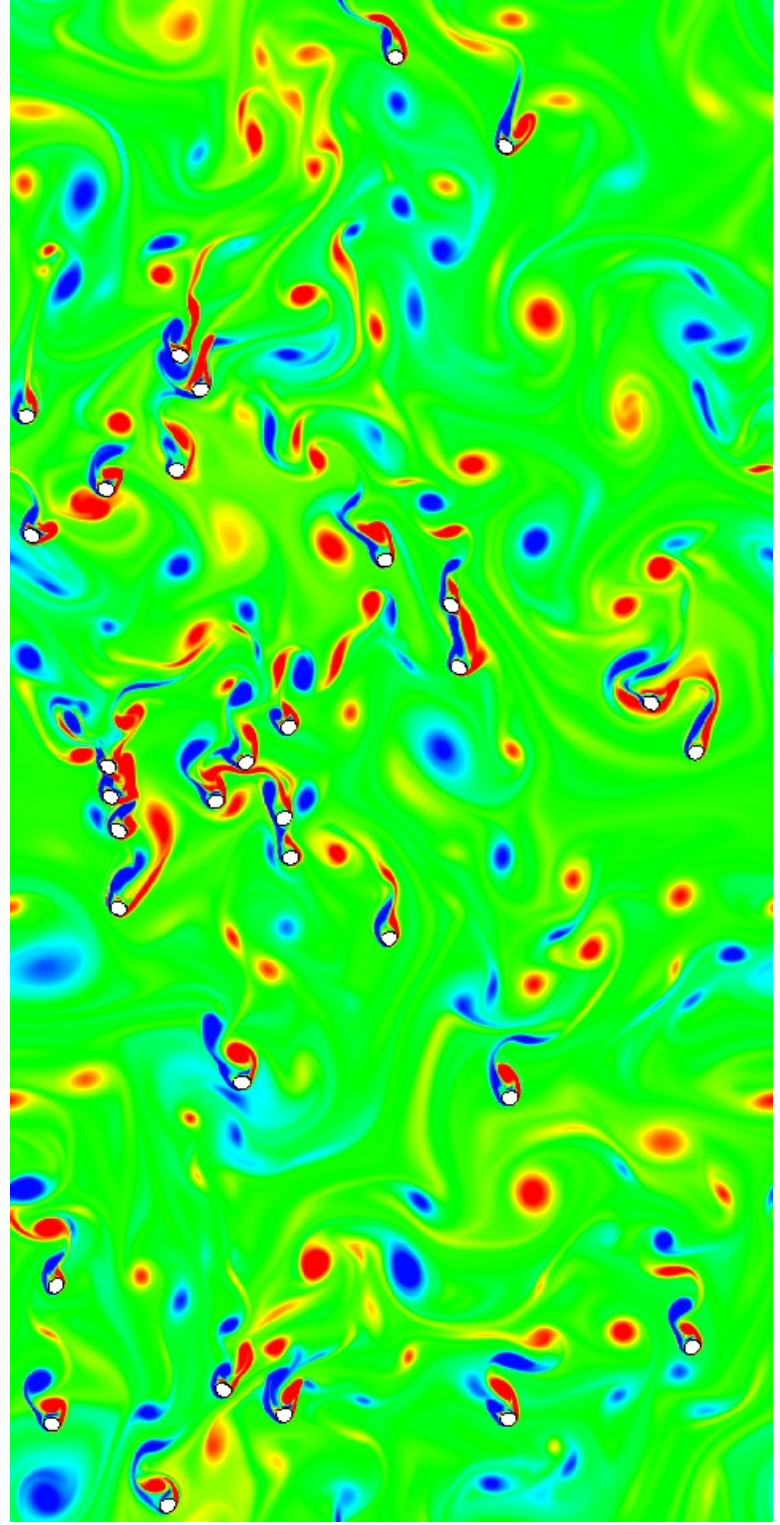

(b) Vortical structures for Case 1 with $E^{*}=1$ and $n=$ 1.2

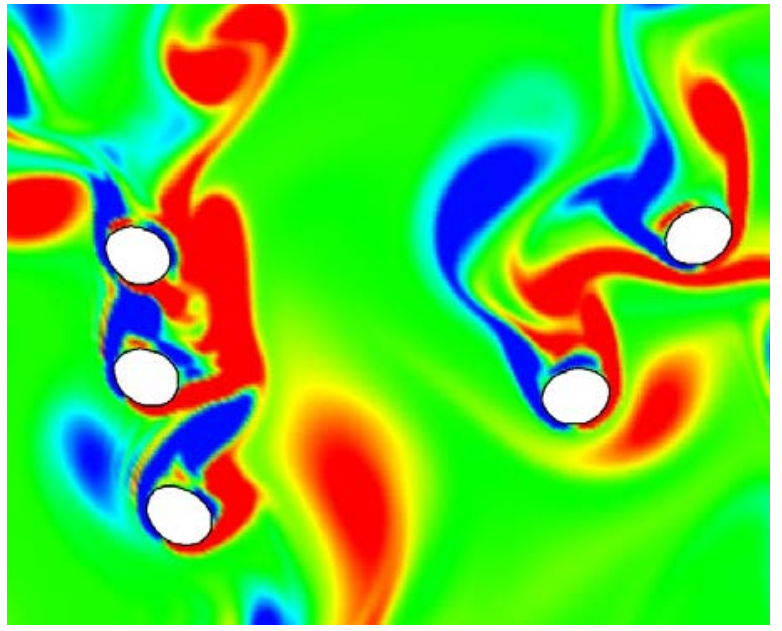

(d) A close-up image of (b)

Figure 4: Elastic particle sedimentation in an up-current flow. (a)(b) Particle distribution and vortical structures visualised by total vorticity $(|\boldsymbol{\omega}|)$ for ellipticity $n=1,1.2$, respectively . (c)(d) Close-up view of clustered behaviours of particles and interaction with vortical structures for ellipticity $n=1,1.2$, respectively. 
Table 1: Simulation conditions for flow field and elastic particles

\begin{tabular}{|cc||c|c|}
\hline & & Case 1 & Case 2 \\
\hline Domain size & $L_{x} \times L_{y}$ & $51.2 D_{p} \times 102.4 D_{p}$ & $102.4 D_{p} \times 204.8 D_{p}$ \\
\hline Spatial resolution & $D_{p} / \Delta$ & 20 \\
\hline Time increment & $\Delta t$ & \multicolumn{2}{|c|}{$2.5 \times 10^{-3}$} \\
\hline Reynolds number & $R e$ & \multicolumn{2}{|c|}{200} \\
\hline Num. of particles & $N_{p}$ & 32 & 128 \\
\hline Sld. vol. fraction & $\varepsilon_{p}$ & \multicolumn{2}{|c|}{$0.48 \%$} \\
\hline Ellipticity & $n$ & $1,1.2$ \\
\hline Norm. Young modulus & $E^{*}=E / \rho U_{0}^{2}$ & \multicolumn{2}{|c|}{$1,10,100$} \\
\hline Mass density ratio & $\rho_{p} / \rho_{f}$ & 2.5 \\
\hline
\end{tabular}

vortices with colour contour. Figures 4(c) and 4(d) are the enlarged images of typical vortical discharge from the clustered particles; some particles are observed to be trapped into the wake vortices of the descending particles, forming a vertical beads structure. Those particles descend as a pair/assembly at a faster speed than a single particle. However, as the minimum inter-particle distance is controlled by the particle-particle potential [10], the behaviours of tumbling and kissing of the particles are not observed at the typical interval and duration $[44,45]$. Observation of the subsequent snapshots shows that the circular particles do not take specific orientation, while the elliptic particles fall swinging around the most stable position (with the major axis pointing the horizontal direction).

Figure 5 shows some sequential snapshots of the flow fields induced by the $n=1$ and $E^{*}=1$ particles, visualised by the contour of the vertical component of the flow field; red and blue regions are (relatively) upand down-flowing regions, respectively. Development of an adjacent up/down-current (coherent streaky) structure is confirmed. Figure 6 is also an instantaneous flow field for the case with 128 circular particles of $E^{*}=1$ in a four-times larger computational domain (Case 2). A larger scale of coherent flow structure is found. However, no clear streaky structure was observed for the elliptic cases as far as we tested.

Figure 7 shows, for the case employing 128 particles, diagrams of average particle Reynolds number $\overline{R e_{p}}$ defined with average slip velocity, root mean square of angular velocities $\operatorname{RMS}\left(\boldsymbol{\omega}_{p}\right)$ and average distance to most neighbouring particle $\overline{L_{p}}$. Smaller $\overline{L_{p}}$ means that dense population may be formed. Effects of non-circular geometry and deformability are studied in the following.

\subsubsection{Effect of non-circularity geometry}

The average particle Reynolds number $\overline{R e_{p}}$ in Fig.7(a) are larger than the particle Reynolds number of a single falling particle $(R e=200)$. A vertical beads structure is heavier than a single particle, but the drag force mainly works on the leading particle, resulting in a faster descent velocity. Our previous study also observed the similar phenomena [46]. 


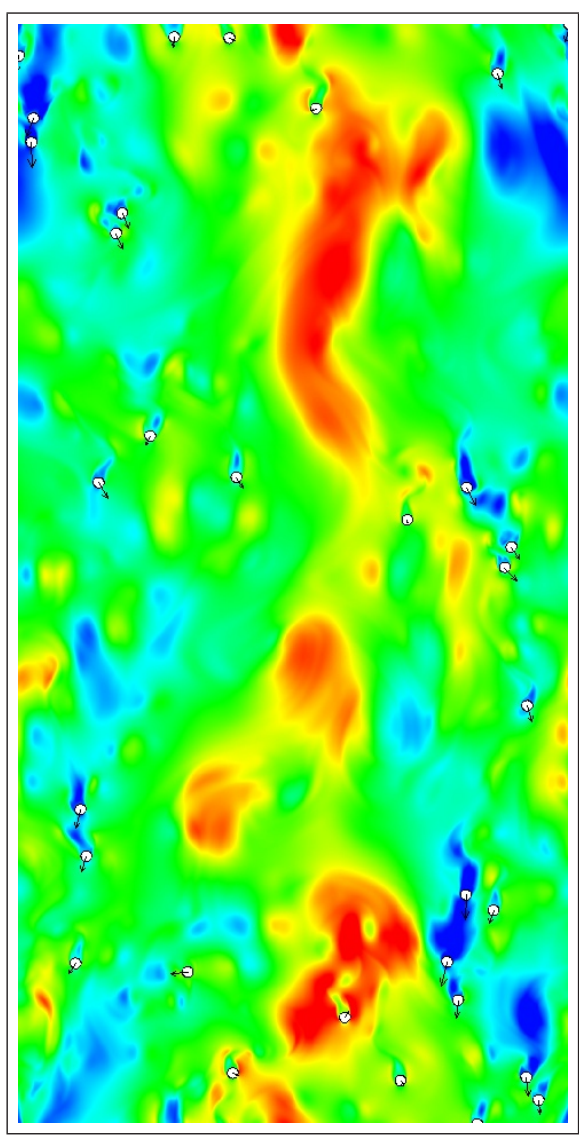

(a) $t=425$

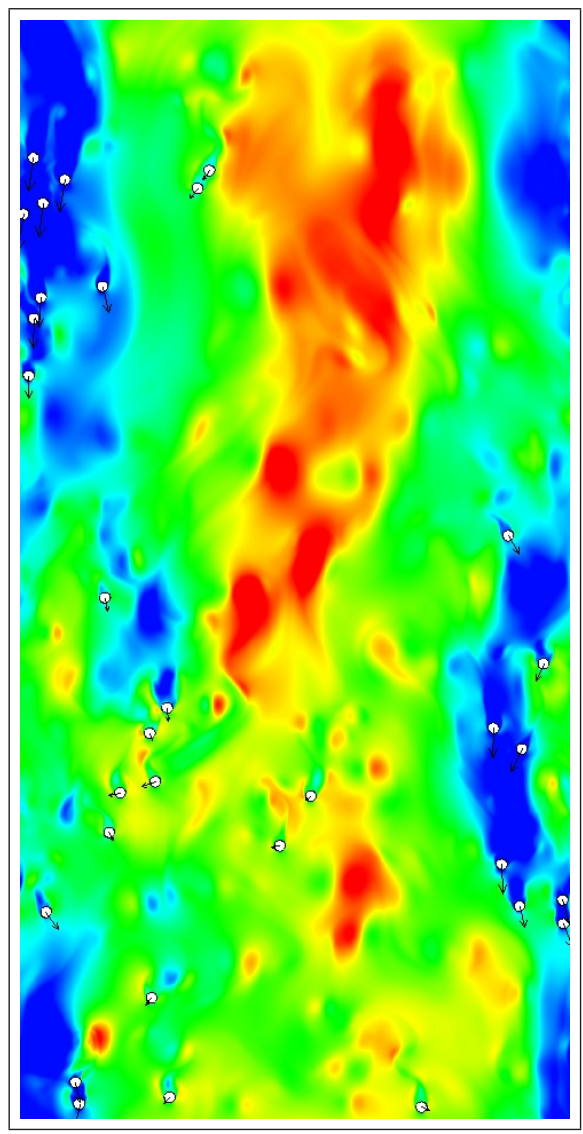

(c) $t=475$

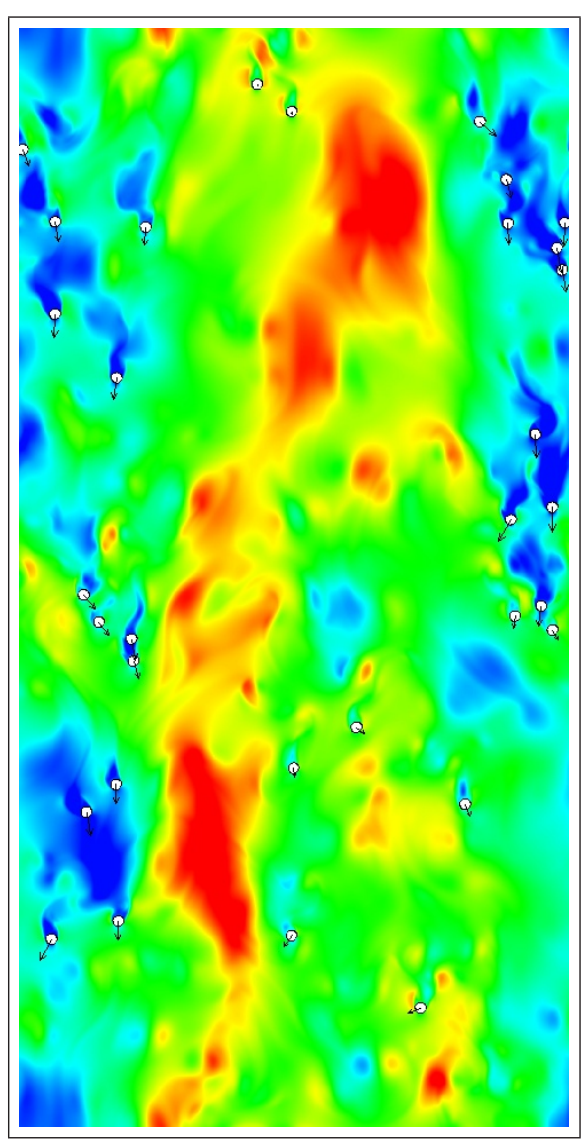

(b) $t=450$

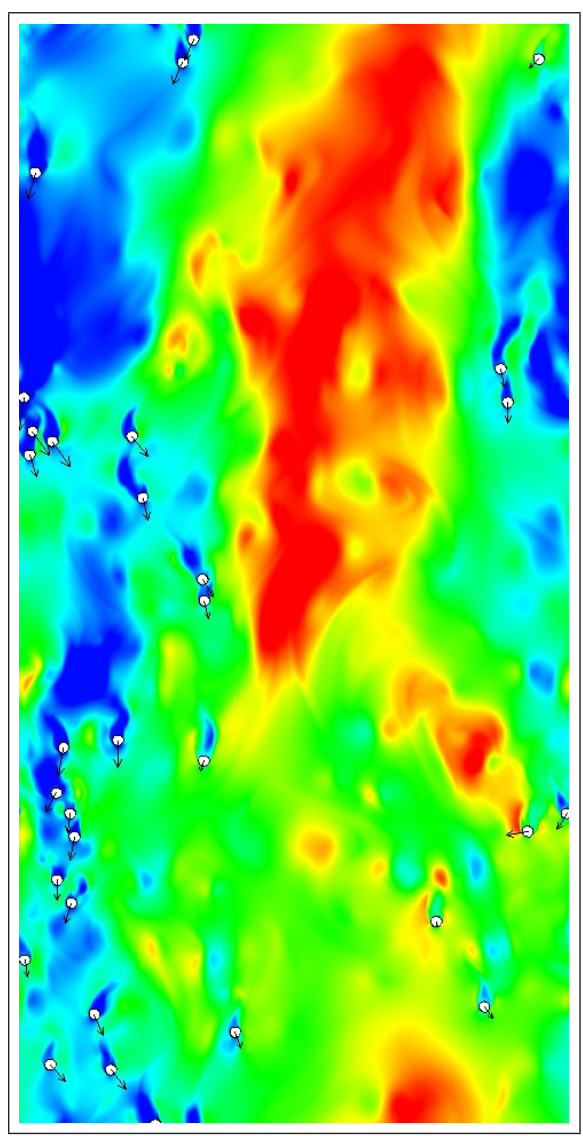

(d) $t=500$

Figure 5: Particle distribution (white) and flow structure coloured by the level of vertical component of the fluid velocity. $n=1, E^{*}=1, t=425$ to 500 . 


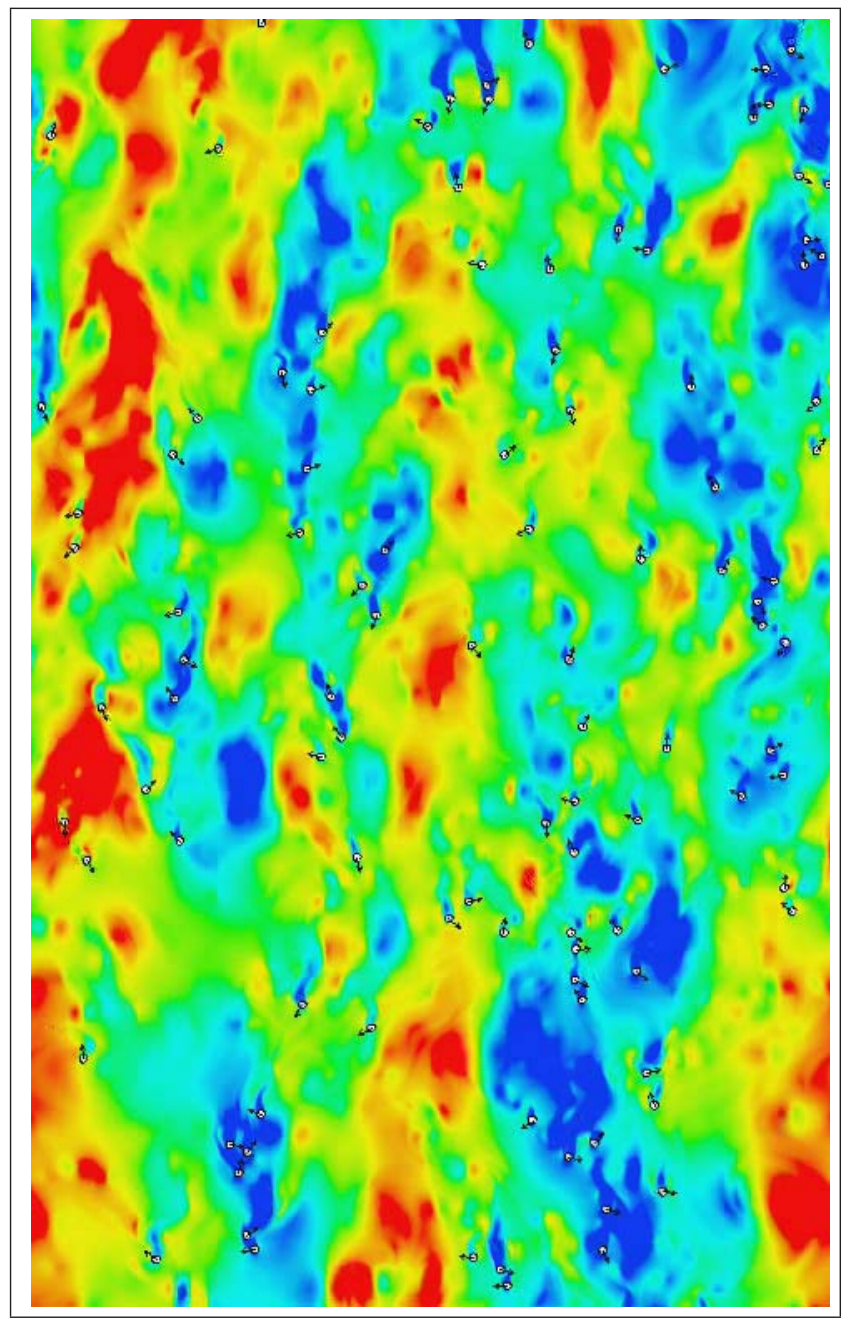

Figure 6: Snapshot of clustering behaviour of 128 elastic particles particles (white) for circular- $E^{*}=1$ case and the coherent flow structures coloured by the level of the vertical component of the fluid velocity. 


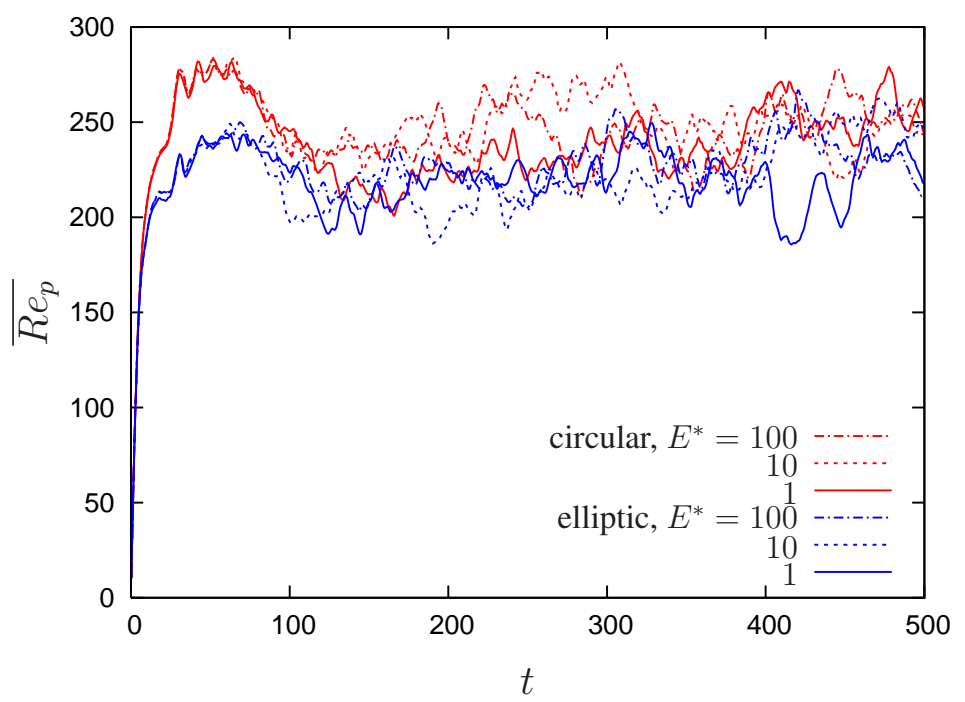

(a) Particle Reynolds number

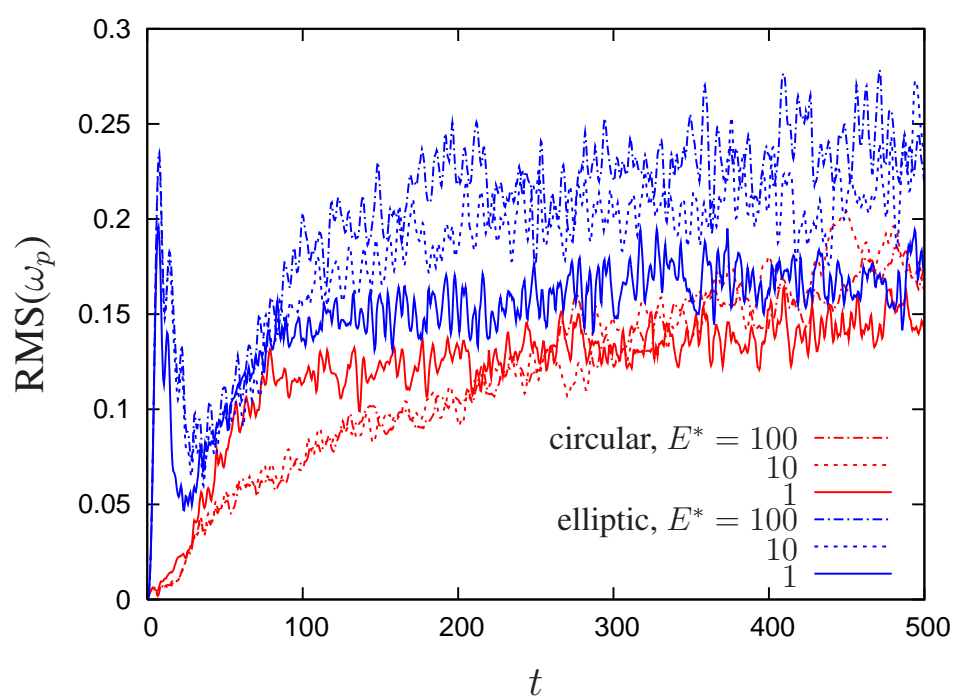

(b) Root mean square (rms) of angular velocity $\omega_{p}$

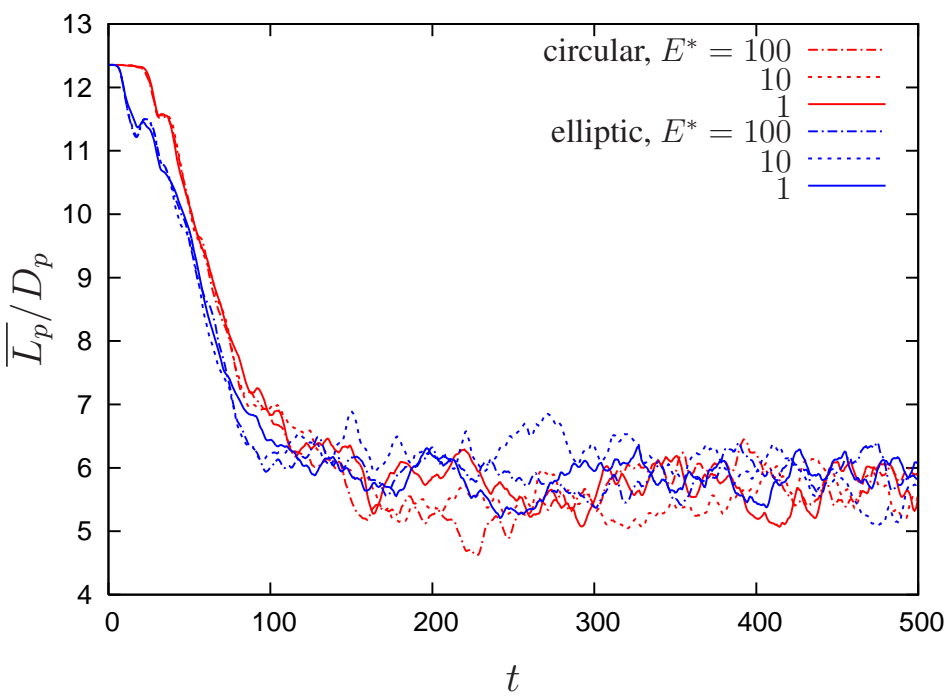

(c) Mean value of inter-particle distance

Figure 7: Statistics of the flow and the particles for different neutral shapes and elasticities in Case 2 (the larger domain and $N_{p}=128$ ). Time evolutions of (a) particle Reynolds number, (b) root mean square of angular velocity and (c) mean value of the closest inter-particle distance. 
The elliptic particles also shows tumbling motion. However, the elliptic particles have the preferred posture, which results in the effective increase in projected area against the descent direction and, in turn, enhancement in drag force. This trend is insensitive to the Young moduli tested. Therefore, the increase in average Reynolds number is slightly lower than the circular cases.

In Fig.7(b), the levels of the angular velocities are obviously different for elliptic and circular particles, especially for the cases with the hard material. Circular particles fall staggering due to the time-varying lift force caused by the vortical discharges. However, rotating motion of (deformed) circular object is affected little by pressure drag (form drag), but mainly by the viscous drag.

For elliptic particles, on the other hand, non-uniform pressure distribution around a particle becomes a major contributing factor for rotating motion. When an elliptic particle rotates, it has to displace the fluid and the first order moment of restitution force works to damp the rotating motion. Therefore, the swinging motions of the elliptic particles exhibit the sudden changes in rotating motion in a short period of time. Figure 7(b) also suggests the strong intensity of fluctuation of angular velocities for the three elliptic cases.

Figure 7(c) shows that, after $t=150$, the smallest $\overline{L_{p}}$ values are attained by the circular particles (regardless of the elasticities). The present author [46] has reported that spherical particles of disabled rotating mobility tend to form clusters (dense population zones). The above result suggests that the correlation between the rotation mobility and clusterisation may also apply to non-circular particles.

The formation process of dense population zone for the soft circular particles is also confirmed from Fig.5. In the figure, the arrows attached to the particles indicate the particle velocities. When a vertical column of up-flowing region is formed in the middle of the domain at $t=425$, the particles are rather distributed unevenly in either side of the up-flow region. As the time goes, the nests of the particles are formed in the down-flow region. When relatively up- and down-flow regions clearly divide the domain vertically at $t=500$, some columnar structures of the particles are formed.

\subsubsection{Effect of deformability}

For elliptic particles of $E^{*}=1$, rotating motions can be further damped than the harder ellipses since the drag force is consumed for deformation as well as rotation. This is also confirmed from Fig.7(b): $E^{*}=1$ ellipses exhibit effectively smaller RMS $\left(\boldsymbol{\omega}_{p}\right)$ values.

Cumulative probability density functions of the presence of the particles are shown in Fig. 8 as a function of the distance from a particle. The probability functions are averaged over the total number of particles in the domain. The obvious difference exists in the particle-presence probability for the two different types of the soft particles. The graph also suggests that the soft circular particles tend to form a nest of particles, whereas the soft non-circular particles have less number of particles in the neighbourhood. 


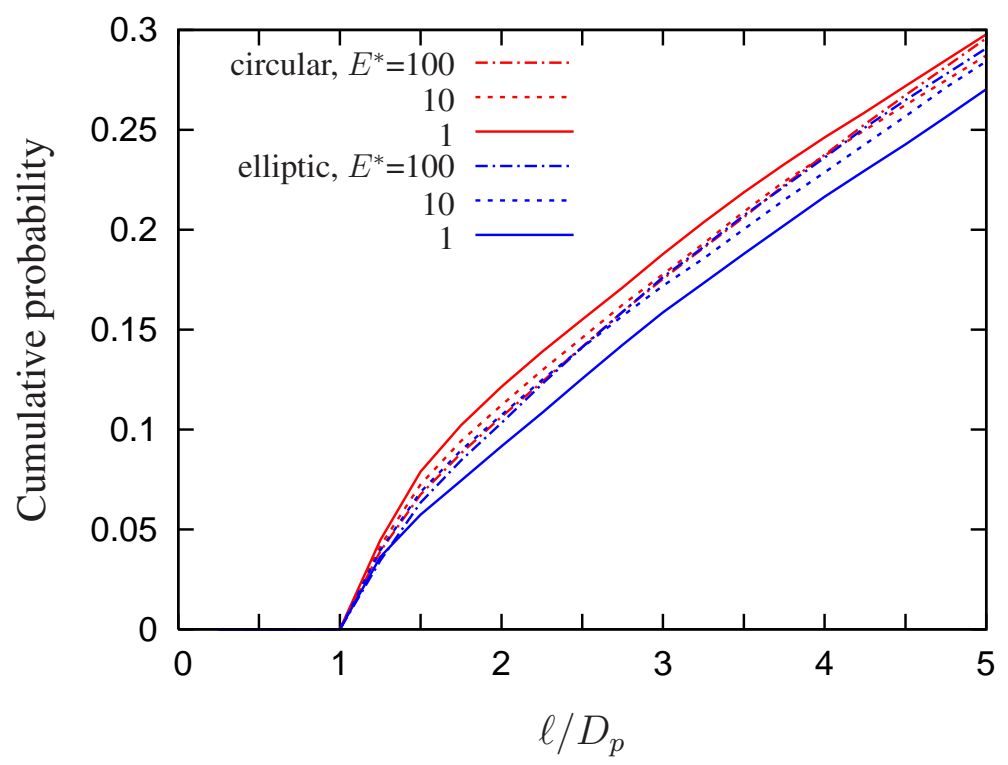

Figure 8: Cumulative probability density profiles of particle presence within the distance $\ell$ from a target particle for different neutral shapes and elasticities in Case 2 (the larger domain with $N_{p}=128$ ).

The above findings suggests that the effect of deformability manifests itself on the motion of particles and clustering behaviour when the deformable nature is added to non-circular objects.

Figure 9 shows stress distributions in circular and elliptic particles visualised by von Mises stress. Elasticity $E^{*}$ is 1 for both cases. The particles are stressed most at the leading edges in the descending directions. It was also observed, in the cases of $E^{*}=1$ and 10 , the tumbling particles exhibit characteristic shapes depending on the places from the leading particles. The effect of deformability on the particle shape and the clustering behaviour is the subject of ongoing research by the present authors.

\section{Concluding Remarks}

A numerical method was developed to solve interaction problems between a fluid and elastic particles. Elastic property of the solid material was incorporated into the fluid dynamics by combining a finite element (FE) method with our finite-difference(FD)-based immersed boundary technique.

The applicability of the mixed FD-FE approach with the momentum-exchange algorithm is demonstrated through simulating multiple elastic particles falling in a 2-D flow field, and the effects of particle geometry and elasticity on the flow structure and clustering behaviour of the particles were studied.

Difference in neutral shape of the particles (circular/non-circular) was found to affect the rotating behaviours of the particles due to the difference in resistance against rotation. Also, the circular objects tend to form dense-population region (cluster) in comparison with the non-circular object. 


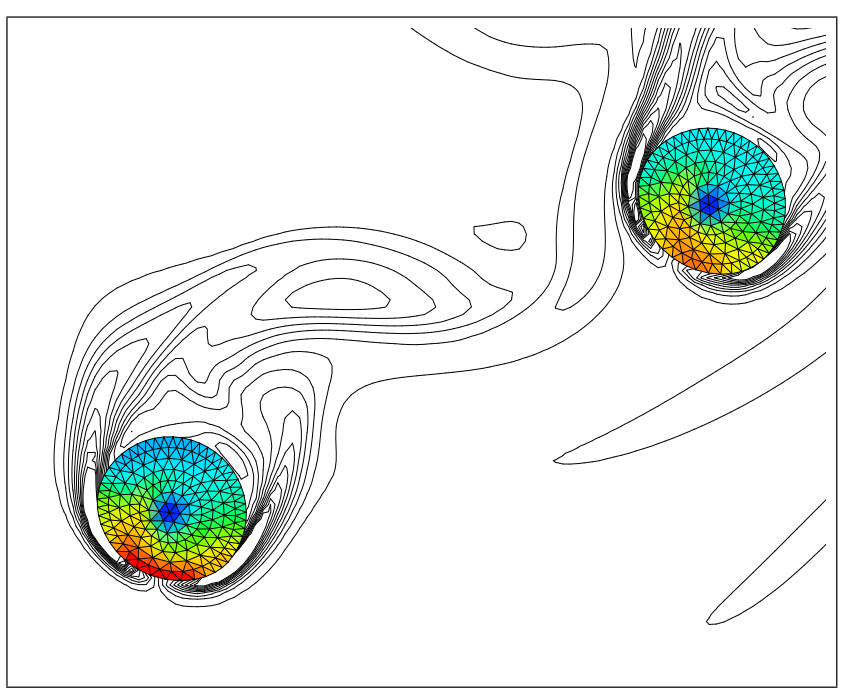

(a) Circular particles

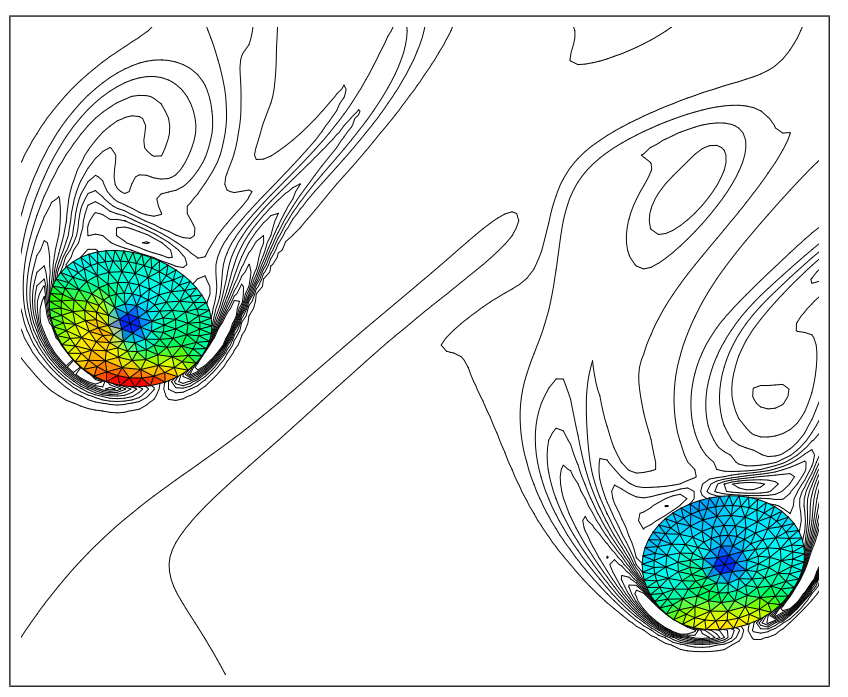

(b) Elliptic particles

Figure 9: Distribution of von Mises stress in the particles of $E^{*}=1$ and the surrounding flow fields visualised by iso-contours of vorticity.

Given some deformability to the non-circular objects, on the other hand, the resistance against rotation abated, while the deformability of the particles showed less possibility of cluster formation for soft noncircular particles in the fluid.

The results suggest that the behaviours of the particles can be strongly influenced by the deformability of the particles, indicating that incorporating non-circularity as well as deformability to solid particles could be an important aspect for deeper understanding of the particle-laden flow, particularly for biological applications.

The present mixed FD-FE approach combined with our momentum-exchange algorithm successfully presented to enable analysis of the interaction problem between a fluid flow and a large number of deformable objects. The method promises to be a unique engineering tool available for many challenging problems, especially many-body problems involving fluid and multiple elastic objects.

The present simulation adopts some assumptions for the feasibility of the study. Considering more practical applications of fluid-deformable-objects interaction problems, geometric nonlinearity with a range of solid materials needs to be incorporated. A numerical tool for this purpose has been established with some solidified validation [39, 40], and more detailed analysis of the clustering behaviour and characteristic deformation of the particles are the subject of a future paper by the present authors. 


\section{References}

[1] Fischer, T.M., Stöhr-Liesen, M. \& Schmid-Schönbein, H., "The red cell as a fluid droplet: tank treadlike motion of the human erythrocyte membrane in shear flow" Science 202, pp.894-896 (1978)

[2] Seifert, U., "Configurations of fluid membranes and vesicles", Advances in Physics 46, pp.13-137 (1997)

[3] Stein, K., Benney, R., Tezduyar, T. \& Potvin, J., "Fluid-structure interactions of a cross parachute: numerical simulation", Computer Methods in Applied Mechanics and Engineering 191, pp.673-687 (2001)

[4] Tezduyar, T.E., Sathe, S., Pausewang, J., Schwaab, M., Christopher, J. \& Crabtree J., 'Fluid-Structure Interaction Modeling of Ringsail Parachutes”, Computational Mechanics 43, pp.133-142 (2008)

[5] Terashima, H. \& Fujii, K., "Influence of Stores on the Transonic Flutter on a Delta Wing Configuration", AIAA Journal 45-1, pp.237-246 (2007)

[6] Hirt, C.W., Amsden, A.A. \& Cook, J.I., "An Arbitrary Lagrangian-Eulerian Computing Method for All Flow Speeds" Journal of Computational Physics 14, pp.227-253 (1974)

[7] Tezduyar, T.E., Behr M. \& Liou, J., "A new strategy for finite element computations involving moving boundaries and interfaces - The deforming-spatial-domain/space-time Procedure: I. The concept and the preliminary numerical tests." Computer Methods in Applied Mechanics and Engineering 94, pp.339-351 (1992)

[8] Tezduyar, T.E., Behr, M., Mittal, S. \& Liou J., "A New Strategy for Finite Element Computations Involving Moving Boundaries and Interfaces - The Deforming-Spatial-Domain/Space-Time Procedure: II. Computation of Free-surface Flows, Two-liquid Flows, and Flows with Drifting Cylinders", Computer Methods in Applied Mechanics and Engineering 94, pp.353-371 (1992)

[9] Peskin, C.S., "Flow patterns around heart valves: a numerical method", Journal of Computational Physics 10-2, pp.252-271 (1972)

[10] Glowinski, R., Pan, T.W., Helsa, T.I., Joseph, D.D. \& Périaux, J., ”A ficticious domain approach to the direct numerical simulation of incompressible viscous flow past moving rigid bodies: application to particulate flow", Journal of Computational Physics 169, pp.363-426 (2001)

[11] Hu, H.H., "Direct Simulation of Flows of Solid-liquid Mixtures", International Journal of Multiphase Flow 22, pp.335-352 (1996)

[12] Johnson, A.A. \& Tezduyar, T.E., "Simulation of Multiple Spheres Falling in a Liquid-filled Tube", Computer Methods in Applied Mechanics and Engineering 134 pp.351-373 (1996)

[13] Mittal, S. \& Tezduyar, T.E., "Parallel Finite Element Simulation of 3D Incompressible Flows-FluidStructure Interactions", International Journal for Numerical Methods in Fluids 21, pp.933-953 (1995) 
[14] Liu, H., Wassersug, R.J. \& Kawachi, K., "A Computational Fluid Dynamics Study of Tadpole Swimming”, Journal of Experimental Biology 199, pp.1245-1260 (1996)

[15] Zhang, Q. \& Hisada, T., "Analysis of fluid-structure interaction problems with structural buckling and large domain changes by ALE finite element method", Computer Methods in Applied Mechanics and Engineering 190-48, pp.6341-6357 (2001)

[16] Saksono, P.H., Dettmer, W.G. \& Perić, D. "An Adaptive Remeshing Strategy for Flows with Moving Boundaries and Fluid-Structure Interaction", International Journal for Numerical Methods in Engineering 71-9, pp.1009-1050 (2007)

[17] Gao, T. \& Hu, H.H., "Deformation of elastic particles in viscous shear flow", Journal of Computational Physics 228, pp.2132-2151 (2009)

[18] Johnson, A.A. \& Tezduyar, T.E., "Advanced Mesh Generation and Update Methods for 3D Flow Simulations", Computational Mechanics 23, pp.130-143 (1999)

[19] Johonson, A. \& Tezduyar, T., "3D simulations of fluid-particle interactions with the number of particles reaching 100", Computer Methods in Applied Mechanics and Engineering 145, pp.301-321 (1997)

[20] Uhlmann, M., "Investigating turbulent particulate channel flow with interface-resolved DNS", Proceedings of the International Conference on Multiphase Flow (ICMF2007), Paper No. S3_Tue_A_16, Leipzig, Germany, 9-13 July, 2007

[21] Peskin, C.S., "The immersed boundary method", Acta Numerica 11, pp.479-517 (2003)

[22] Gilmanov, A. \& Sotiropoulos, F., "A hybrid Cartesian/immersed boundary method for simulating flows with 3D, geometrically complex, moving bodies", Journal of Computational Physics 207, pp.457-492 (2005)

[23] Eggleton, C.D. \& Popel, A.S., "Large deformation of red blood cell ghosts in a simple shear flow", Physics of Fluids 10, pp.1834-1845 (1998)

[24] Zhang, L.T. \& Gay, M., "Immersed Finite Element Method for Fluid-Structure Interactions", Journal of Fluids and Structures 23-6, pp.839-857 (2007)

[25] Gay, M. \& Zhang, L.T., ”Imposing Rigidity Constraints on Immersed Objects in Unsteady Fluid Flows", Computational Mechanics 42, pp.357-370 (2008)

[26] LeVeque, R.J. \& Li, Z., "The immersed interface method for elliptic equations with discontinuous coefficients and singular sources", SIAM Journal on Numerical Analysis 31, pp.1019-1044 (1994)

[27] Li, Z. \& Lai, M.-C., "The Immersed Interface Method for the Navier-Stokes Equations with Singular Forces", Journal of Computational Physics 171, pp.822-842 (2001)

[28] Tan, Z., Lim, K.M. \& Khoo, B.C., "An Immersed Interface Method for Stokes Flows with Fixed/Moving Interfaces and Rigid Boundaries", Journal of Computational Physics 228, pp.68556881 (2009) 
[29] Kajishima, T., Takiguchi, S., Hamasaki, H. \& Miyake, Y., "Turbulence structure of particle-laden flow in a vertical plane channel due to vortex shedding", JSME International Journal Series B 44-4, pp.526-535 (2001)

[30] Kajishima, T. \& Takiguchi, S., "Interaction between particle clusters and fluid turbulence", International Journal of Heat and Fluid Flow 23(5), pp.639-646 (2002)

[31] Yuki, Y., Takeuchi, S. \& Kajishima, T., "Efficient Immersed Boundary Method for Strong Interaction Problem of Arbitrary Shape Object with the Self-Induced Flow" Journal of Fluid Science and Technology 2-1, pp.1-11 (2007)

[32] Nishiura, D., Shimosaka, A., Shirakawa, Y. \& Hidaka, J., "Hybrid Simulation of Hindered Settling Behavior of Particles Using Discrete Element Method and Direct Numerical Simulation" (in Japanese), Kagaku Kogaku Ronbunshu (Trans. Soc. Chem. Eng. Japan) 32-4, pp.331-340 (2006)

[33] Cundall, P.A. \& Strack, O.D.L., "A Discrete Numerical Model for Granular Assembles", Geotechnique 29, pp.47-65 (1979)

[34] Amsden, A.A. \& Harlow, F.H., "A simplified MAC technique for incompressible fluid flow calculations", Journal of Computational Physics 6, pp.322-325, 1970

[35] Tamura, A., Kikuchi, K. \& Takahashi, T., "Residual cutting method for elliptic boundary value problems: Applications to Poisson's equation”, Journal of Computational Physics 137-2, pp.247-264 (1997)

[36] Takeuchi, S., Kajishima, T., Wang, X.S. \& Rhodes, M.J., "Study of Gas-Particle Flow by Direct Numerical Simulations", Proceedings of the 32nd Australian Chemical Engineering Conference (CHEMECA 2004), Paper No.148, Sydney, Australia, 27-29 September, 2004

[37] Marsden, J.E. \& Hughes, T.J.R., Mathematical Foundations of Elasticity, Prentice-Hall (1983)

[38] Zienkiewicz, O.C. \& Tarlor, R.L., The Finite Element Method (6th edn), Butterworth-Heinemann, (2005)

[39] Ueyama, A., Kajishima, T. \& Takeuchi, S., "Analysis of Interaction between Multiple Deformable Particles and a Fluid Flow by Immersed Boundary-Finite Element coupling Method (IB-FEM)", Proceedings of the 15th International Conference on Finite Elements in Flow Problems (FEF09), Tokyo, Japana, 1-3 April, 2009

[40] Takeuchi, S., Kajishima, T., Iwata, R. \& Ueyama, A., "Large Scale Analysis of Multiphase Flow Involving Dispersed Components with Deformable Boundaries by a New Immersed Boundary Technique Coupled with VOF and FEM" Proceedings of the 6th International Symposium on Multiphase Flow, Heat Mass Transfer and Energy Conversion (ISMF), Xi' an, China, July 11-15, 2009, Paper No. MN-11 (2009)

[41] Ishihara, D. \& Yoshimura, S., "A monolithic approach for interaction of incompressible viscous fluid and elastic body based on fluid pressure Poisson equation", International Journal for Numerical Methods in Engineering 64, pp.167-203 (2005) 
[42] Smith, I. M. \& Griffiths, D.V., Programming the Finite Element Method (4th edn), Wiley (2004)

[43] Uhlmann, M., "An immersed boundary method with direct forcing for the simulation of particulate flows", Journal of Computational Physics 209-2, pp.448-476 (2005)

[44] Tsuji, T., Narutomi, R., Yokomine, T., Ebara, S. \& Shimizu, A., "Unsteady three-dimensional simulation of interactions between flow and two particles", International Journal of Multiphase Flow 29, pp.1431-1450 (2003)

[45] Sharma, N. \& Patankar, N.A., "A fast computation technique for the direct numerical simulation of rigid particulate flows", Journal of Computational Physics 205, pp.439-457 (2005)

[46] Kajishima, T., "Influence of particle rotation on the interaction between particle clusters and particleinduced turbulence", International Journal of Heat and Fluid Flow 25-5, pp.721-728 (2004)

\section{Acknowledgements}

One of the authors, S.T., gratefully acknowledges the financial support of Suzuki Foundation (Tokyo, Japan) for the modelling of coupling between a fluid and deformable object. He is also grateful for the Kurata Memorial Hitachi Science and Technology Foundation (Tokyo, Japan) for sponsoring for the analysis of clustering behaviour of deformable objects in a fluid. 